\title{
Review
}

\section{Toward an Integrated Understanding of the Purkinje Fibers in the Heart: The Functional and Morphological Interconnection between the Purkinje Fibers and Ventricular Muscle}

\author{
Hideo Tanaka ${ }^{1}$, Tetsu Hamamoto ${ }^{1,2}$ and Tetsuro Takamatsu ${ }^{1}$ \\ ${ }^{1}$ Department of Pathology and Cell Regulation, Kyoto Prefectural University of Medicine, Kamigyo-ku, Kyoto 602-8566, Japan \\ and ${ }^{2}$ Department of Cardiovascular and Respiratory Medicine, Shiga University of Medical Science, Otsu, Japan
}

Received June 1, 2005; accepted July 26, 2005

\begin{abstract}
Purkinje fibers are an essential element of the conduction system that provides for coordinated ventricular contraction of the heart. Although classic studies established structure and electrical activities of Purkinje fibers, an integrated understanding of the Purkinje-ventricular interconnection within the working heart remains to be had. In this review article we will briefly overview the gross anatomy and cytological characteristics of Purkinje fibers and their transition to the ventricular muscle, and we will discuss

functional and morphological aspects of the Purkinje-ventricular interconnection from the perspectives of electrophysiology, distribution of gap junctions, and intracellular $\mathrm{Ca}^{2+}\left(\left[\mathrm{Ca}^{2+}\right]_{i}\right)$ dynamics. Furthermore, the potential usefulness of the recently developed optical voltagemapping and in situ real-time confocal $\left[\mathrm{Ca}^{2+}\right]_{i}$ imaging techniques is discussed as an integrated approach to the pathophysiological investigation of the Purkinje-ventricular interconnection in the heart.
\end{abstract}

Key words: Purkinje fiber, conduction, imaging, confocal microscope, arrhythmia

\section{Introduction}

The Purkinje fibers of the heart constitute a complex network of excitable cells that orchestrates the rhythmic and coordinated ventricular contraction for efficient ejection of blood to the circulation $[8,35]$. This specialized tissue was discovered by Purkinje in 1845 , who first identified it macroscopically as a net of gray, flat, gelatin-like fibers on the endocardial surface of the ventricles [32]. During the following century much was clarified about the precise structure and functions of the Purkinje fibers. However, most morphological and functional studies were conducted respectively with fixed, serial sections and preparations excised mainly from the papillary muscles [52]. Therefore, no integrated understanding was gained about the structure

Correspondence to: Hideo Tanaka, M.D., Ph.D., Department of Pathology and Cell Regulation, Kyoto Prefectural University of Medicine, Kamigyo-ku, Kyoto 602-8566, Japan

E-mail: hideotan@koto.kpu-m.ac.jp and functions of the Purkinje fibers within the heart in situ. Recent advances in functional imaging technologies $[9,11]$ and their application to genetically engineered animals [29] have paved the way for elucidating the functional and morphological correlation between the Purkinje-fiber network and its underlying ventricular myocardium in more detail. In this review article, we discuss the progress in studies on Purkinje fibers, with special reference to their interconnection with the ventricular myocardium.

\section{Gross Anatomy of Purkinje Fibers}

The conduction system of the ventricles originates from the bundle of His followed by right- and left-bundle branches, and extends to the periphery of the subendocardial tissue as the Purkinje fibers that connect with the subjacent ventricular myocardium $[8,28,35]$. The detailed anatomical basis of this specialized conduction system was established by Tawara [45], who demonstrated in 1906 extensive branching of the fiber network on the subendocardial 

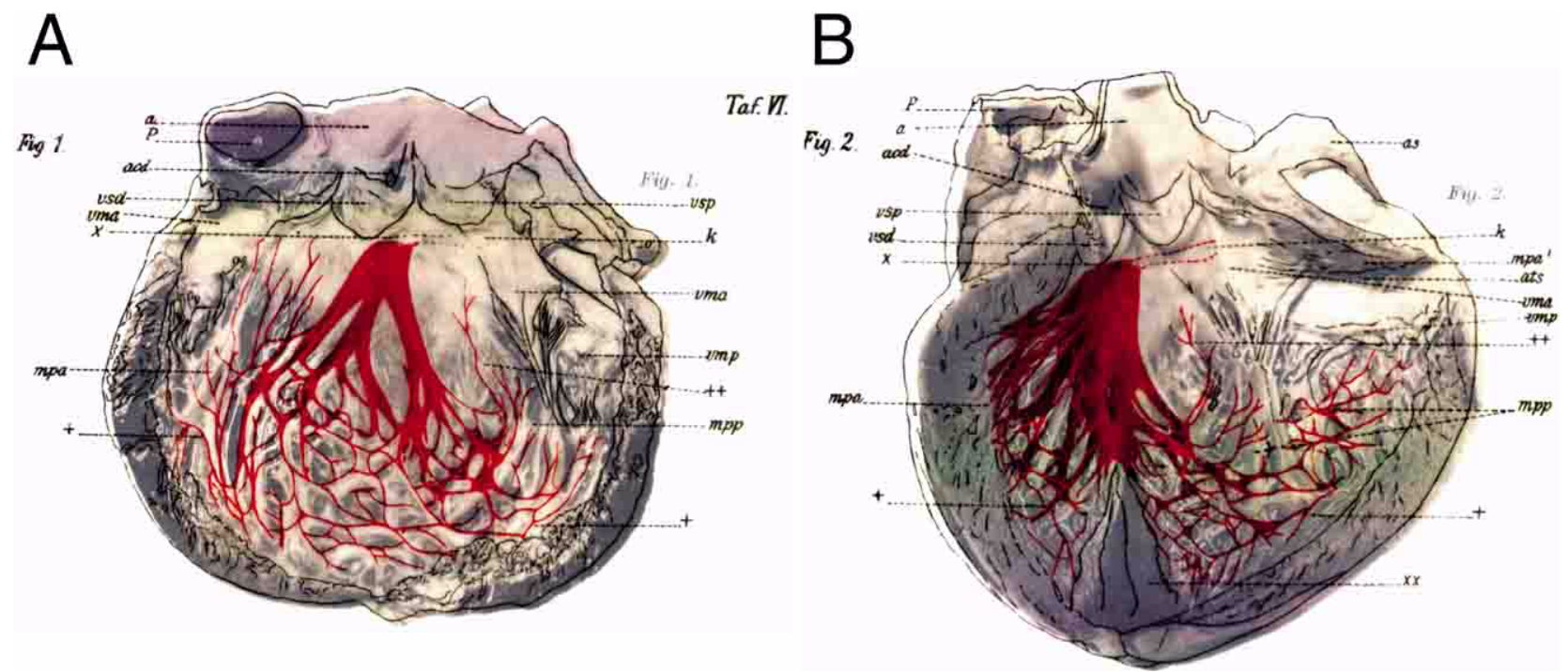

Fig. 1. Drawings of the His-Purkinje systems scanned from the Tawara's monograph [45]. Panel (A) shows branching of the left bundle and the following Purkinje-fiber network (in red) within the subendocardial layer. Panel (B) shows the right bundle branch and the Purkinje network (in red).

layer by reconstruction from serial sections (Fig. 1). However, the sections for histological specimens allowed visualization of only a limited number of large branches, and therefore the terminal ramifications of Purkinje fibers were not traced precisely due to their complex anatomy.

Subsequent investigators established a method for tracing peripheral Purkinje fibers to the terminal ramification by direct injection of India ink into the Purkinje fibers $[1,19,48,52]$. This method revealed that the Purkinje fibers were distributed extensively as a subendocardial network, forming a polygonal arrangement with many separate fascicles surrounded by a perifascicular connective tissue sheath until their extension to the ventricular myocytes. Before reaching the ventricular myocytes the Purkinje fibers cross, ramify, and anastomose each other, thereby forming a threedimensional network (Fig. 2A) [1].

\section{Cytological Properties of Purkinje Fibers}

The fundamental structural difference between the Purkinje fibers and ventricular myocytes resides in their respective absence and presence of transverse (T)-tubules [38]. Even in the large (or proximal) Purkinje fibers no Ttubular system is detected. Therefore, while the sarcoplasmic reticulum (SR) of the ventricular myocyte efficiently couples with the sarcolemma of the T-tubule (interior coupling) and with the peripheral sarcolemma (peripheral coupling), the Purkinje fibers form only peripheral couplings with the SR.

The Purkinje fibers are specifically stained by periodic acid Schiff (PAS) reaction, whereas ventricular muscle fiber is not $[16,51]$. This is because glycogen is more abundant in the conduction systems than in the ordinary myocytes [54]. The high glycogen content of Purkinje fibers indicates that the fibers metabolize the glycogen with anaerobic enzymes, providing greater resistance to hypoxia than is found in the ventricular myocardium [10].

Acetylcholine esterase activity is detected in the conduction system but not in the ventricular myocardium [5]. Histochemical detection of the esterase activity by acetylthiocholine iodide (ATCHI) staining visualized the thin right-bundle branch in the mouse heart [42]. The peripheral Purkinje fibers are also stained with ATCHI in the rat heart (Fig. 2B).

\section{Fate of the Peripheral Purkinje Fibers}

The junctions between the terminal Purkinje fibers and subjacent ventricular muscle (hereafter, $\mathrm{P}-\mathrm{V}$ junctions) are electrophysiologically well-defined entities, where subendocardial ventricular activation begins with a delay on the order of $5 \mathrm{~ms}$ after excitation of Purkinje fibers [24]. In principle, the P-V junctions reside in the subendocardial layer along their course to several fiber ramifications which contact with the subjacent ventricular fibers [52]. MartinezPalomo and Benitez [23] in 1970 found that the terminal Purkinje fibers in dog heart are followed by cells of a transitional type which make contact with the subjacent ventricular fibers. They characterized the transitional cells by (a) their subendocardial location, (b) their small diameter, (c) their absence of T-tubular systems, (d) their higher glycogen content, (e) their lack of clearly defined intercalated discs, and (f) absence of branching. Toshimori et al. [46] demonstrated in pig heart that Purkinje fibers are immunoreactive to atrial natriuretic peptide (ANP), whereas the transitional region between the Purkinje fibers and the ventricular myocytes is weakly stained and the ventricular myocytes are not stained. Scanning electron microscopic images from goat 
A a

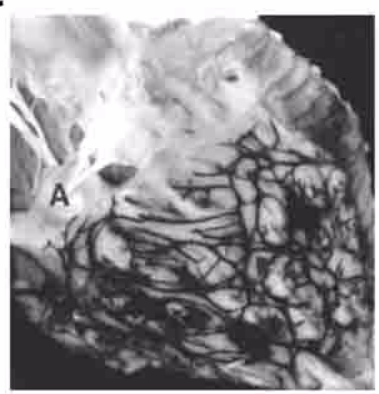

b

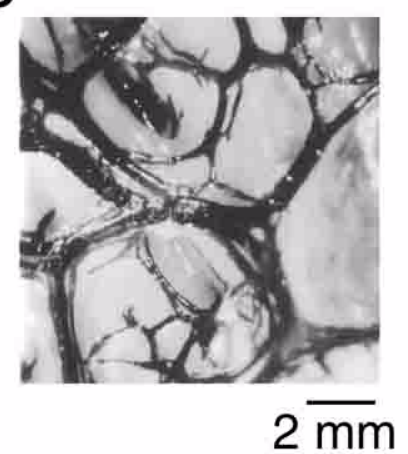

B

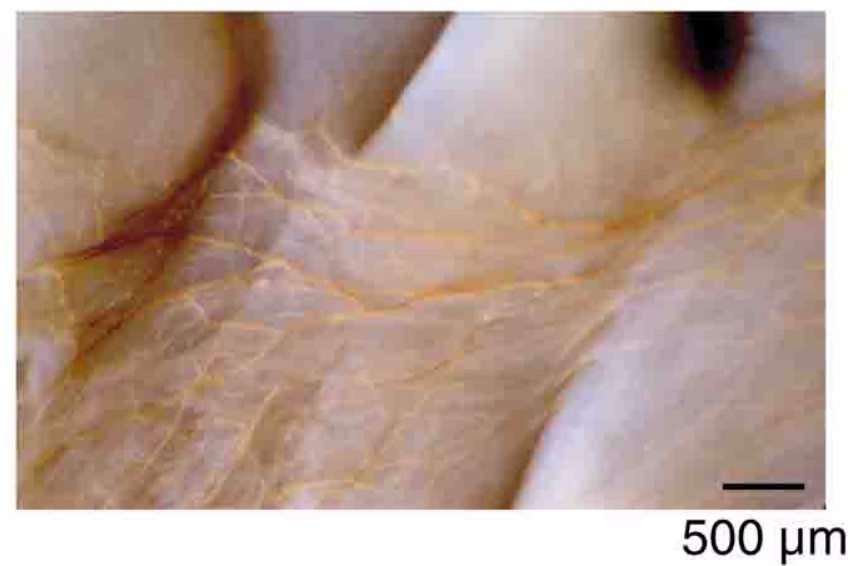

$\mathrm{Ca}$
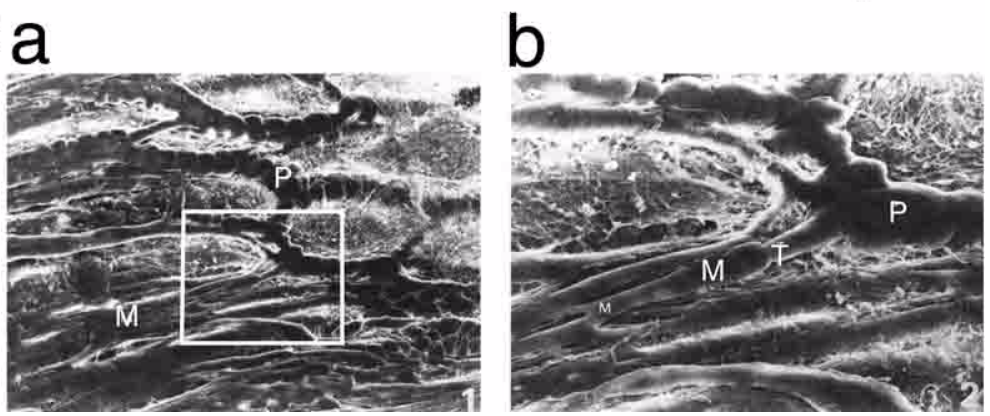

Fig. 2. A: Purkinje fibers visualized by injection of India ink. (a) Purkinje-fiber network in the subendocardial layer of the right ventricle. Note the polygonal arrangement in the subendocardium showing extensive branching and anastomosis (b). Reproduced from Ansari et al. [1] with permission. B: Dissecting microscopic image of Purkinje fibers in the papillary muscle of the right ventricular septum of the rat heart stained with ATCHI. C: Scanning electron micrograph of the transitional cells of the sheep subendocardial network (a) and a close-up view (b) of the ruled area in (a). Purkinje fibers (P) show a network, which transforms to ordinary ventricular myocytes (M). There are transitional cells (T) between $\mathrm{P}$ and M. Reproduced from Shimada et al. [36] with permission.

heart also distinguish the transitional cells at the P-V junctions from the neighboring cells [36] (Fig. 2C). Electrophysiologically, the transitional cells mediate impulse propagation of the Purkinje fibers to the ventricular myocytes in rabbits and pigs [47], and are regarded as sites of highresistance coupling between Purkinje fibers and ventricular myocardium. Such a resistive barrier between the two tissues allows for rapid propagation to the Purkinje-fiber layer on the endocardial surface and synchronized activation throughout the ventricles. However, no definite transitional cells are identified in some species; in human and bovine hearts, the Purkinje fibers gradually become smaller and eventually become similar to the ventricular myocytes at the junctions without precisely recognizable transitional cells [30].

The Purkinje fibers not only distribute on the subendocardial surface but also penetrate the ventricular muscle for varying distances, especially in large mammals [28]. These intramural fibers are arranged as a complex threedimensional network and likely contribute to a more efficient excitation of the ventricles compared with the absence of intramural fibers. 


\section{Electrophysiological Aspects of Purkinje- Ventricular Muscle Interconnection}

The functional interconnection between Purkinje fibers and ventricular muscles has been extensively studied by microelectrode recordings of the action potentials mainly from excised preparations of papillary muscles [8, 24-26, 28]. These studies revealed that the P-V junctions have a relatively low safety factor for electrical impulse propagation, i.e., propagation between these two tissues can easily be impaired due to the relatively poor cell-to-cell coupling at the junction [50]. Conduction disturbance at $\mathrm{P}-\mathrm{V}$ junctions can occur under various pathological states, especially under ischemic conditions, e.g., hypoxia, hyperkalemia, and acidosis [12]. Impairment of electrical coupling at the $\mathrm{P}-\mathrm{V}$ junction provides an opportunity to create a reentrant circuit of excitation, an important substrate for the development of ventricular tachyarrhythmias [21, 34, 39]. Microelectrode recording also suggested that ventricular arrhythmias in acute ischemia arise from ectopic focal excitation within the Purkinje-fiber network of the peri-infarct zone [17]. In spite of these electrophysiological studies, precise spatiotemporal information on the activation sequence was lacking on the Purkinje-ventricular interconnection. Although recent electrical mapping techniques of the whole heart $[2,6,9,42]$, especially fluorescent imaging with voltage-sensitive dyes [42], have provided spatiotemporal patterns of ventricular activation, the spatial resolution of such activation mapping systems still does not permit accurate delineation of the activation pattern of the conduction system at the Purkinje-fiber network with high spatial resolution, e.g., at subcellular levels. Thus, the functional and morphological correlation of the conduction system has yet to be established in the heart in situ.

\section{Connexin Mediation of Conduction in Purkinje Fibers}

Propagation of the electrical impulse is much more rapid in Purkinje fibers $(2-3 \mathrm{~m} / \mathrm{s})$ than in ventricular muscle $(0.3-0.4 \mathrm{~m} / \mathrm{s})$ [8]. Such differences in propagation velocity can in part be explained by the differences of intercellular coupling via connexins, a family of proteins forming gap junctions, which are important in determining impulse propagation in the heart [31]. Three distinct types of connexins, connexin40 (Cx40), connexin 43 (Cx43), and connexin 45 $(\mathrm{Cx} 45)$, are mainly involved in impulse propagation of the heart. Of these, $\mathrm{Cx} 43$ is a major gap junction protein in the ventricles, whereas $\mathrm{Cx} 40$ is predominantly expressed in the conduction systems and atria $[13,14,18]$. Enriched Cx40 distribution in Purkinje fibers can offer less resistance to current transfer than in the ventricles, which contain more Cx43 [18], and therefore provide a faster conduction velocity in Purkinje fibers than in ventricular muscle.

As a critical determinant of cell-to-cell communication and electrical impulse conduction, the potential role of $\mathrm{Cx} 40$ in the conduction system was evidenced by genetically engi- neered studies, i.e., null mutation of $\mathrm{Cx} 40$ gene. Simon et al. [37] and Kirchhoff et al. [20] demonstrated that the HisPurkinje system in Cx40 knockout (Cx40-/-) mice (Fig. 3) showed uncoordinated ventricular excitation and spatially altered propagation through the working myocardium. Analogously, Tamaddon et al. [42] also demonstrated that high-resolution optical mapping of the right bundle branch in the Cx40-/- mouse reveals slow conduction in the conduction system. Currently, it remains to be determined to what extent the peripheral Purkinje fibers and P-V junction contribute to the slowing of impulse conduction in the Cx40-/- mice.

Besides Cx40-mediated interconnection among Purkinje fibers, distribution of connexins at the P-V junctions is also an important subject of research. At the junction, heterotypic $\mathrm{Cx} 40-\mathrm{Cx} 43$ channels, which consist of two different hemichannels, are postulated to contribute to the propagation of the electrical impulse in the heart [49]. The intercellular coupling resistance of the $\mathrm{Cx} 40-\mathrm{Cx} 43$ heterotypic channels is suggested to be higher than that of the homotypic $\mathrm{Cx} 43$ channels.

\section{Intracellular $\mathrm{Ca}^{2+}$ Dynamics of Purkinje Fibers}

Besides the electrical activities, intracellular $\mathrm{Ca}^{2+}$

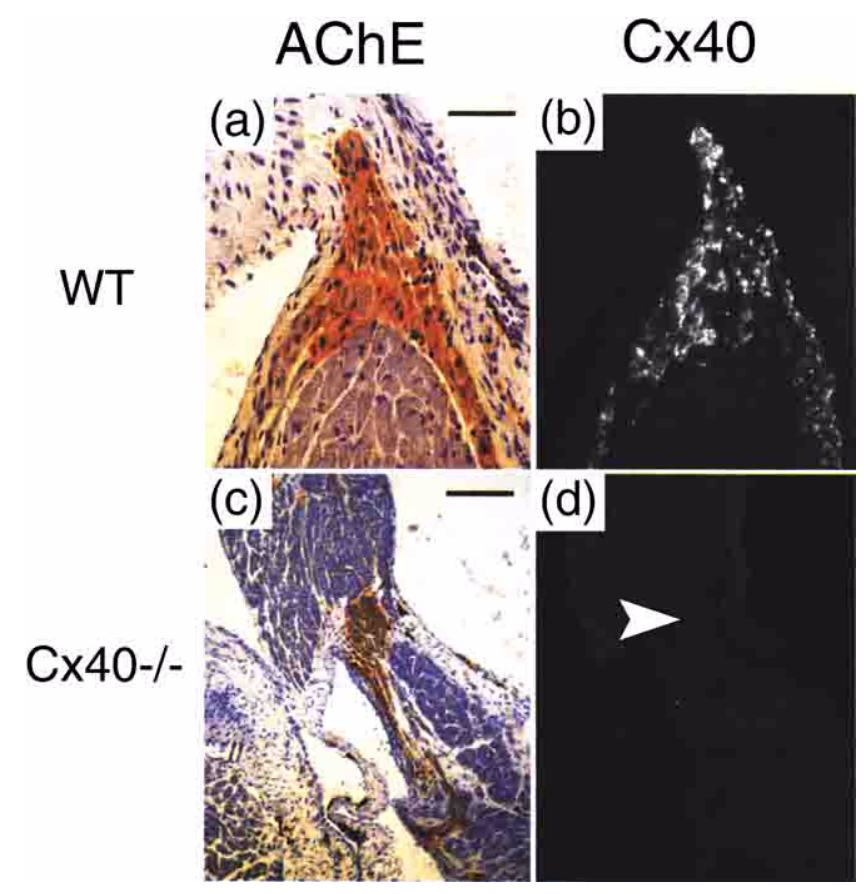

Fig. 3. Connexin expression in the His-Purkinje system in wild type (WT) and connexin40 knockout (Cx40-/-) mice. (a, c) Purkinje fibers were distinguished by acetylcholine esterase (AChE) activity (brown). Cx40 immunoreactivity is remarkable in Purkinje fibers of WT heart (b), whereas no $\mathrm{Cx} 40$ was detected in AChE-positive fibers from $\mathrm{Cx} 40-/-$ animals (d, arrowhead). Bars $=50 \mu \mathrm{m}(\mathbf{a}), 150$ $\mu \mathrm{m}$ (c). Reproduced from Simon et al. [37] with permission. 

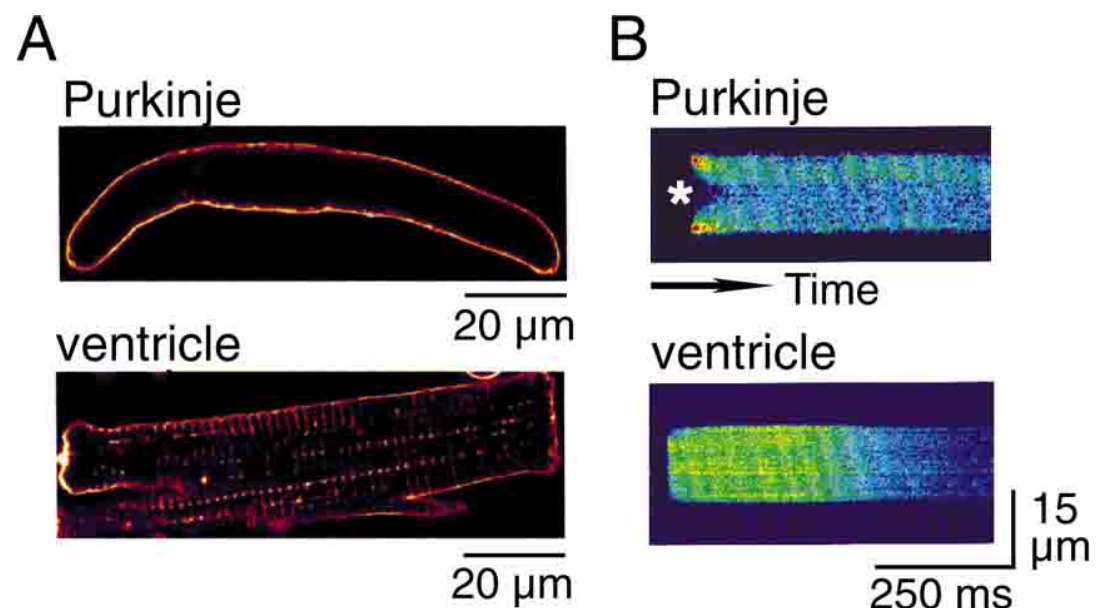

Fig. 4. Comparison of cell structure and $\left[\mathrm{Ca}^{2+}\right]_{i}$ dynamics between a Purkinje fiber and ventricular myocyte of the rabbit. Sarcolemmal staining of Purkinje fiber and ventricular myocytes with di-8-ANEPPS (A). Note that the Purkinje fiber is stained with di-8-ANEPPS only at the periphery of the fiber, indicating the absence of T-tubules, whereas the striations in ventricular myocytes indicate T-tubular system. Representative transverse line-scan images of $\mathrm{Ca}^{2+}$-dependent fluo3-fluorescence intensity from the Purkinje fiber and a ventricular myocyte (B). Note that the ventricular myocyte exhibits spatially homogeneous rise of $\mathrm{Ca}^{2+}$, whereas the Purkinje fiber initiates $\mathrm{Ca}^{2+}$ transients at the periphery and propagates toward the center of the cell as indicated by asterisk. Reproduced from Cordeiro et al. [7] with permission.

$\left(\left[\mathrm{Ca}^{2+}\right]_{\mathrm{i}}\right)$ of the myocardial tissue is also important in determining the impulse generation and propagation of the heart [3]. Confocal microscopy with high spatiotemporal resolution has enabled us to visualize the precise $\left[\mathrm{Ca}^{2+}\right]_{\mathrm{i}}$ dynamics of the heart muscle cells [11]. In the ventricular myocytes, excitation-contraction coupling mediates influx of $\mathrm{Ca}^{2+}$ through the sarcolemmal $\mathrm{Ca}^{2+}$ channels, which triggers the release of $\mathrm{Ca}^{2+}$ from the SR [3]. Since the SR is tightly coupled to sarcolemmal membranes that distribute throughout the T-tubules, the ventricular myocytes exhibit spatially homogeneous $\mathrm{Ca}^{2+}$ transients under physiological conditions. On the other hand, $\mathrm{Ca}^{2+}$ transients in Purkinje fibers initiate at the periphery of the sarcolemma, and subsequently propagate to the center of the fibers because of the lack of T-tubules [7] (Fig. 4). Under pathological conditions, such coordinated $\left[\mathrm{Ca}^{2+}\right]_{i}$ dynamics in individual Purkinje fibers are impaired. For example, enzymatically isolated Purkinje-fiber aggregates from the peri-infarcted heart exhibit spatially inhomogeneous $\left[\mathrm{Ca}^{2+}\right]_{\mathrm{i}}$ dynamics showing spontaneous micro $\mathrm{Ca}^{2+}$ transients or intracellular propagation of $\left[\mathrm{Ca}^{2+}\right]_{\text {i }}$ (i.e., $\mathrm{Ca}^{2+}$ waves) [4] similar to those in injured ventricular myocytes [41, 43, 44].

\section{In Situ Interconnection of $\mathrm{Ca}^{2+}$ Dynamics at the P-V Interface}

Having established the precise morphology in the ventricular conduction system, the next focus is the functional and morphological interconnection of the Purkinje-ventricular interface in the heart in situ. The confocal $\left[\mathrm{Ca}^{2+}\right]_{\mathrm{i}}$ dynamics at the subendocardial surface of the heart delineates the precise spatiotemporal activation patterns of the Purkinje fibers and the underlying ventricular myocytes [15]. This approach reveals that the Purkinje-fiber network contrib- utes to a syncytial function of the heart in conducting the impulse uniformly to the ventricular myocardium. Purkinje fibers exhibit spatiotemporally coordinated $\mathrm{Ca}^{2+}$ transients in individual fibers, which closely couple with the subjacent ventricular myocytes with no discernible delay in $\mathrm{P}-\mathrm{V}$ activation, indicating a syncytial function of the Purkinjefiber network in delivering the impulse to the ventricles (Fig. 5A). Such spatiotemporal synchrony of $\mathrm{Ca}^{2+}$ transients would suggest a mechanism to prevent inhomogeneous conduction from the Purkinje network to the ventricles leading to micro-reentrant excitation. Nevertheless, a unidirectional block can occur at P-V junctions. Hyperkalemia, a major cause of intraventricular conduction disturbances in ischemic hearts [53], produces various patterns of conduction disturbances between Purkinje fibers and ventricular myocytes. Elevation of $\mathrm{K}^{+}$concentration from $5.4 \mathrm{mM}$ to $10 \mathrm{mM}$ produced an orthodromic Purkinje-to-ventricular block (a), whereas an antidromic, ventricle-to-Purkinje conduction was preserved at $10-\mathrm{mM} \mathrm{K}^{+}$concentration (b), i.e., a unidirectional block at $\mathrm{P}-\mathrm{V}$ junctions (Fig. 5B). This may lead to macroscopic reentrant excitation among different branches of the network, which could eventually induce reentrant tachyarrhythmias.

Abnormal $\left[\mathrm{Ca}^{2+}\right]_{i}$ dynamics reflecting cardiac pathology are also detected in the Purkinje fiber network with in situ confocal microscopy. As shown in Figure 6, intracellular propagation of wave-like $\left[\mathrm{Ca}^{2+}\right]_{\mathrm{i}}$ (i.e., $\mathrm{Ca}^{2+}$ waves) emerges in presumably injured fibers (e.g., p1-p3), similar to the $\mathrm{Ca}^{2+}$ waves in ventricular myocytes. In addition, some Purkinje fibers exhibit spontaneous $\mathrm{Ca}^{2+}$ transients that are dyssynchronous to the intrinsic heart rhythm (e.g., p5). These abnormal $\left[\mathrm{Ca}^{2+}\right]_{\mathrm{i}}$ dynamics may represent intra- and intercellular bases for abnormal excitation and conduction at the Purkinje-fiber network leading to ventricular tachy- 




Fig. 5. $\left[\mathrm{Ca}^{2+}\right]_{\mathrm{i}}$ dynamics in Purkinje-fiber network of the right ventricular septum. Sequential $\mathrm{X}-\mathrm{Y}$ images (with frame numbers on the upper left) (A) of the fluo3-fluorescence in Purkinje fibers (a) and the surrounding ventricular myocardium (b). The images shown were taken at 66-ms intervals. Line-scan images (d) were obtained from the scan lines on Purkinje fibers (p1 and p2) and a ventricular myocyte (v) shown in (c). Corresponding electrocardiogram (ECG) is on the bottom. B: Changes in $\mathrm{Ca}^{2+}$ transients induced by high- $\mathrm{K}^{+}(10 \mathrm{mM})$ perfusion. Line-scan images of Purkinje fibers (p1-p3) and the underlying ventricular myocytes (v1-v3) are shown. Corresponding electrocardiograms are illustrated on the top. Orthodromic $\mathrm{P}-\mathrm{V}$ conduction is disturbed (i.e., $\mathrm{P}-\mathrm{V}$ block) by $10-\mathrm{mM} \mathrm{K}^{+}$perfusion under atrial pacing at 2-Hz frequency (a), whereas retrograde V-P conduction is preserved (b): $\mathrm{Ca}^{2+}$ transients in Purkinje fibers are evoked by ventricular pacing from the apex (red arrows). Reproduced from Hamamoto et al. [15] with permission.

arrhythmias.

Besides $\left[\mathrm{Ca}^{2+}\right]_{\mathrm{i}}$ dynamics, in situ confocal microscopy also allows us to morphologically distinguish the cell structure of the individual Purkinje fibers from the subjacent ventricular myocytes. Staining of the cell membrane with di-4ANEPPS revealed a network of Purkinje fibers which was remarkably distinguished from the subjacent ventricular myocytes: Purkinje fibers in rat hearts were significantly thinner (approx. $6 \mu \mathrm{m}$ in diameter) than ventricular myocytes $(19 \mu \mathrm{m})$ and were devoid of T-tubules (Fig. 7A). Three-dimensional representation of tissue architecture from the confocal images provides further information [40]. Stereo images of the subendocardial surface of the right ventricular septum clearly visualized 3-dimensional Purkinje-fiber ramification and the subjacent regular alignment of ventricular myocytes (Fig. 7B).

\section{Transgenic Approaches for an Integrated Understanding of Purkinje Fibers}

With the recent progress in research on cardiac development, several specific markers for the conduction system have been discovered [29]. Rentschler et al. [33] created a line of transgenic mouse where the lac $Z$ reporter gene expression delineates the developing and mature murine cardiac conduction system, extending from the sinoatrial node to 


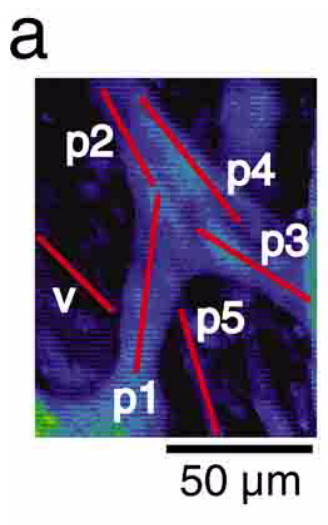

b

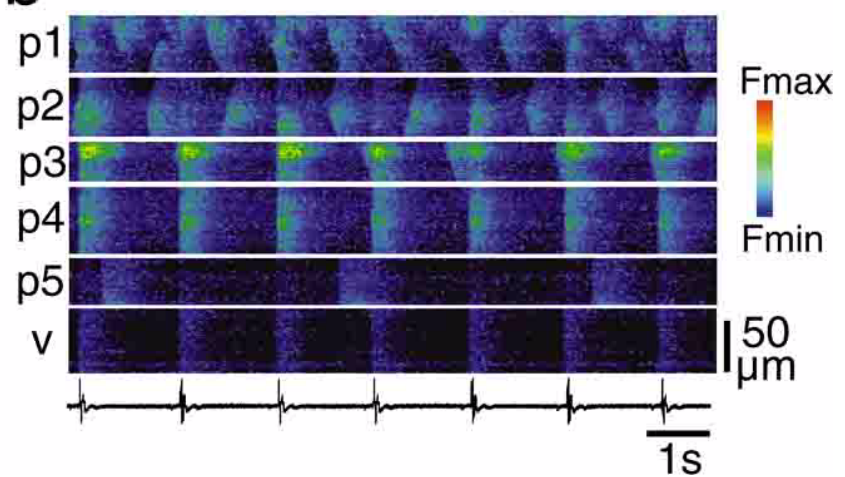

Fig. 6. $\mathrm{Ca}^{2+}$ waves and dyssynchronous $\mathrm{Ca}^{2+}$ transients in the Purkinje-fiber network. X-Y image of a Purkinje-fiber network (a), X-t images of Purkinje fibers (p1-p5) and neighboring ventricular myocytes (v) (b). Electrocardiogram is shown below the X-t images. Note that the Ca ${ }^{2+}$ waves (appear as oblique lines in p1-p3) emerge in Purkinje fibers in between $\mathrm{Ca}^{2+}$ transients. One Purkinje fiber (p5) shows spontaneous $\mathrm{Ca}^{2+}$ transients which are dyssynchronous to the basic heart rhythm. Reproduced from Hamamoto et al. [15] with permission.

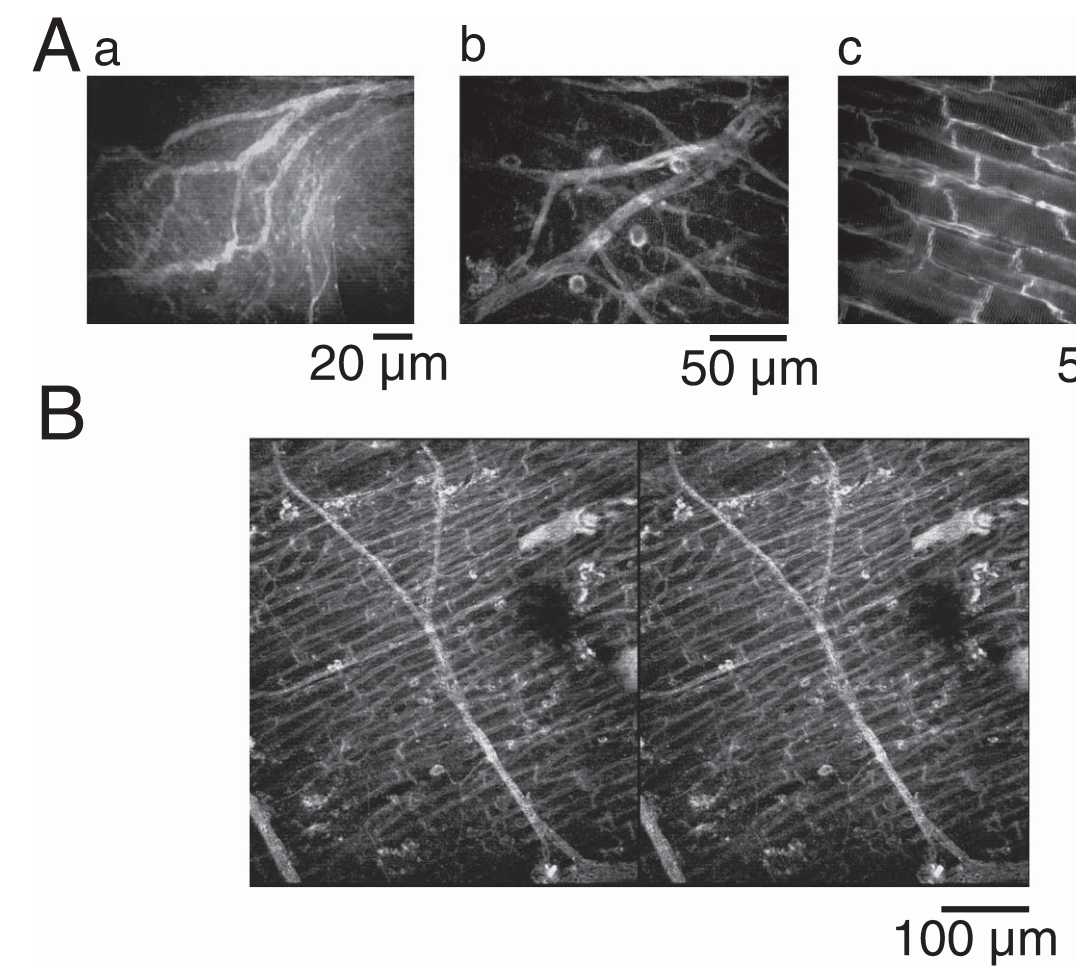

Fig. 7. A: Confocal microscopic images of the Purkinje fibers (a \& b) and subjacent ventricular myocardium (c) stained with di-4-ANEPPS. Reproduced from Hamamoto et al. [15] with permission. B: Stereo images of the subendocardial surface of the right ventricular septum stained with di-4-ANEPPS. Note the 3-dimensional distribution of Purkinje fibers showing ramification and subjacent regular alignment of ventricular myocytes.

the distal Purkinje fibers (Fig. 8A). Optical excitation mapping of the heart using a voltage-sensitive dye confirms that cells identified by the lac $Z$ reporter gene are identical to the components of the specialized conduction system. Kondo et al. [22] also demonstrated that $\beta$-gal staining of embryonic hearts from the $\operatorname{minK}$-lac $Z$ mouse delineates the developing cardiac conduction system. More recently, Miquerol et al. [27] created a transgenic mouse line where EGFP is ex- pressed under the control of the $\mathrm{Cx} 40$ gene $\left(\mathrm{Cx} 40^{\mathrm{EGFP} /+}\right.$ mouse) (Fig. 8B). They identified the His-Purkinje system of the adult mouse heart by EGFP expression and revealed conduction patterns by optical mappings. This mouse model could become a useful tool for simultaneous analysis for morphology and function of the His-Purkinje system. At present, it remains to be determined whether these fluorescent markers are expressed in the peripheral Purkinje fibers 

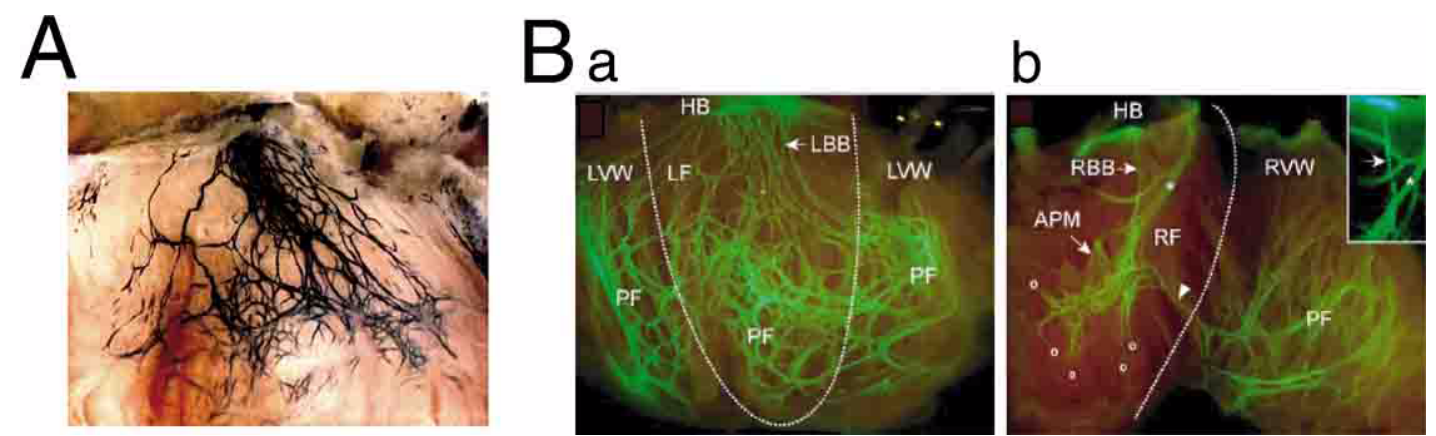

Fig. 8. A: Purkinje-fiber network of the septal surface of the left ventricle in a mouse heart with lacZ-expression of the cardiac conduction system. B: Conduction system of the $\mathrm{Cx} 40^{\mathrm{EGFP} /+}$ mice. EGFP-positive signals are seen in the His-Purkinje system of the left (a) and right (b) ventricular chambers. Reproduced from (A) Myers and Fishman [29], and (B) Miquerol et al. [27] with permission.

and $\mathrm{P}-\mathrm{V}$ junction. If these markers are homogeneously expressed in the Purkinje-fiber network, such transgenic approaches could become a powerful strategy to clarify the precise structural and functional correlation of the P-V junctions.

\section{Future Directions}

Recent optical mapping techniques have revolutionized research on electrical activity and $\left[\mathrm{Ca}^{2+}\right]_{i}$ dynamics of the conduction system and provided deeper insights into the cardiac conduction system [9]. At present, however, the voltage-sensitive dye mapping of the global activation pattern fails to provide clear discrimination between Purkinje fibers and ventricular muscles because of the low spatial resolution. On the other hand, in situ real-time confocal imaging of the conduction system provides precise images for intra- and intercellular $\mathrm{Ca}^{2+}$ dynamics and cellular structures, but not the corresponding electrical activities, due to its relatively low temporal resolution and its difficulty in detecting tiny voltage-sensitive fluorescence. In the next decade, simultaneous application of advanced molecular probes for the membrane voltage and $\left[\mathrm{Ca}^{2+}\right]_{i}$ will enable us to visualize spatially more discriminative information on the conduction system. Combined application of these techniques to the transgenic animals with specific marking for the conduction system or gap junctions will provide us with a more integrated understanding of the physiology and pathology of the Purkinje fibers in the working heart in situ.

\section{Acknowledgments}

This work was supported in part by Grants-in-Aid from the Ministry of Education, Science, Sports, and Culture of Japan.

\section{References}

1. Ansari, A., Ho, S. Y. and Anderson, R. H. (1999) Distribution of the Purkinje fibers in the sheep heart. Anat. Rec. 254; 92-97.

2. Asano, Y., Davidenko, J. M., Baxter, W. T., Gray, R. A. and Jalife, J. (1997) Optical mapping of drug-induced polymorphic arrhythmias and torsade de pointes in the isolated rabbit hearts. J. Am. Coll. Cardiol. 29; 831-842.

3. Bers, D. (2001) Excitation-contraction Coupling and Cardiac Contractile Force. Kluwer Academic Publishers, Dordrecht, The Netherlands.

4. Boyden, P. A., Barbhaiya, C., Lee, T. and ter Keurs, H. E. D. J. (2003) Nonuniform $\mathrm{Ca}^{2+}$ transients in arrhythmogenic Purkinje cells that survive in the infarcted canine heart. Cardiovasc. Res. 57; 681-693.

5. Carbonell, L. M. (1955) Esterases of the conduction system of the heart. J. Histochem. Cytochem. 4; 87-97.

6. Cates, A. W., Smith, W. M., Ideker, R. E. and Pollard, A. E. (2001) Purkinje and ventricular conductions to endocardial activation sequence in perfused rabbit right ventricle. Am. J. Physiol. Heart Circ. Physiol. 281; H490-H505.

7. Cordeiro, J. M., Spitzer, K. W., Giles, W. R., Ershler, P. E., Cannell, M. B. and Bridge, J. H. (2001) Location of the initiation site of calcium transients and sparks in rabbit heart Purkinje cells. J. Physiol. (Lond). 531; 301-314.

8. Durrer, D., van Dam, R. T., Freud, G. E., Janse, M. J., Meijler, F. L. and Arzbaecher, R. C. (1970) Total excitation of the isolated human heart. Circulation 41; 899-912.

9. Efimov, I. R., Nikolski, V. P. and Salama, G. (2004) Optical imaging of the heart. Circ. Res. 95; 21-33.

10. Friedman, P. L., Stewart, J. R., Fenoglio, J. J., Jr. and Wit, A. L. (1973) Survival of subendocardial Purkinje fibers after extensive myocardial infarction in dogs. Circ. Res. 33; 597-611.

11. Fujita, K. and Takamatsu, T. (2002) Real-time in situ calcium imaging with single and two-photon confocal microscopy. In "Confocal and Two-photon Microscopy", ed. by A. Diaspro, Wiley-Liss, Inc., New York, pp. 483-498.

12. Gilmour, R. F., Evans, J. J. and Zipes, D. P. (1984) Purkinjemuscle coupling and endocardial response to hyperkalemia, hypoxia, and acidosis. Am. J. Physiol. 247; H303-H311.

13. Gourdie, R. G., Severs, N. J., Green, C. R., Rothery, S., Germroth, P. and Thompson, R. P. (1993) The spatial distribution and relative abundance of gap-junctional connexin 40 and connexin 43 correlate to functional properties of component of the cardiac atrioventricular conduction system. J. Cell Sci. 105; 985-991.

14. Gross, B. D., Jarry-Guichard, T., Ten Velde, I., de Maziere, A., van Kempen, M. J., Davoust, J., Briand, J. P., Moorman, A. F. and Jongsma, H. J. (1994) Restricted distribution of connexin 40, a gap junctional protein, in mammalian heart. Circ. Res. 74; 839851.

15. Hamamoto, T., Tanaka, H., Mani, H., Tanabe, T., Fujiwara, K., Nakagami, T., Horie, M., Oyamada, M. and Takamatsu, T. (2005) In situ $\mathrm{Ca}^{2+}$ dynamics of Purkinje fibers and its interconnection with subjacent ventricular myocytes. J. Mol. Cell. 
Cardiol. 38; 561-569.

16. Hondeghem, L. M. and Stroobandt, R. (1973) Purkinje fibers of sheep papillary muscle: occurrence of discontinuous fibers. $\mathrm{Am}$. J. Anat. 141; 251-262.

17. Janse, M. J. and Wit, A. L. (1989) Electrophysiological mechanisms of ventricular arrhythmias resulting from myocardial ischemia and infarction. Physiol. Rev. 69; 1049-1169.

18. Kanter, H. L., Laing, J. G., Beau, S. L., Beyer, E. C. and Saffitz, J. E. (1993) Distinct patterns of connexin expression in canine Purkinje fibers and ventricular muscle. Circ. Res. 72; 1124-1131.

19. King, M. R. (1916) The sino-ventricular system as demonstrated by the injection method. Am. J. Anat. 19; 149-177.

20. Kirchhoff, S., Nelles, E., Hagendorff, A., Kruger, O., Traub, O. and Willecke, K. (1998) Reduced cardiac conduction velocity and predisposition to arrhythmias in connexin40-deficient mice. Curr. Biol. 8; 299-302.

21. Kleber, A. G., Rieber, A. G. and Janse, M. J. (1987) Electrical uncoupling and increase of extracellular resistance after induction of ischemia in isolated, arterially perfused rabbit papillary muscle. Circ. Res. 61; 271-279.

22. Kondo, R. P., Anderson, R. H., Kupershmidt, S., Roden, D. M. and Evans, S. (2003) Development of the cardiac conduction system as delineated by minK-lacZ. J. Cardiovasc. Electrophysiol. 14; 383-391.

23. Martinez-Palomo, A., Alanis, J. and Benitez, D. (1970) Transitional cardiac cells of the conduction system of the dog heart. J. Cell Biol. 47; 1-17.

24. Matsuda, K., Kamiyama, A. and Hoshi, T. (1967) Configuration of the transmembrane potential of the Purkinje-ventricular fiber junction and its analysis. In "Electrophysiology and Ultrastructure of the Heart", ed. by T. Sano, J. Mizuhira and K. Matsuda, Grune and Stratton, New York, pp. 177-188.

25. Mendez, C., Mueller, W. J., Merideth, J. and Moe, G. K. (1969) Interaction of transmembrane potentials in canine Purkinje fibers and at Purkinje fiber-muscle junctions. Circ. Res. 24; 361-372.

26. Mendez, C., Mueller, W. J. and Urguiaga, X. (1970) Propagation of impulse across the Purkinje fiber-muscle junctions in the dog heart. Circ. Res. 26; 135-150.

27. Miquerol, L., Meysen, S., Mangoni, M., Bois, P., van Rijen, H. V. M., Abam, P., Jongsma, J., Nargeot, J. and Gros, D. (2004) Architectural and functional asymmetry of the His-Purkinje system of the murine heart. Cardiovasc. Res. 63; 77-86.

28. Myerburg, R. J., Nilsson, K. and Gelband, H. (1972) Physiology of canine intraventricular conduction and endocardial excitation. Circ. Res. 30; 217-243.

29. Myers, D. C. and Fishman, G. I. (2004) Toward an understanding of the genesis of murine cardiac pacemaking and conduction system development. Anat. Rec. A. Discov. Mol. Cell. Evol. Biol. $280 ; 1018-1021$

30. Oosthoek, P. W., Viragh, S., Lamers, W. H. and Moorman, A. F. M. (1993) Immunohistochemical delineation of the conduction system. II. The atrioventricular node and Purkinje fibers. Circ. Res. 73; 482-491.

31. Page, E. and Manjunath, C. K. (1986) Communicating junctions between cardiac cells. In "The Heart \& Cardiovascular System", ed. by H. A. Fozzard, E. Haba, R. B. Jennings, A. M. Katz and H. E. Morgan, Ravens Press, New York, pp. 573-560.

32. Purkinje, J. (1845) Mikroskopisch-neurologische Beobachtungen. Arch. Anat. Physiol. u Wiss Med. 12; 281-295.

33. Rentschler, S., Vaidya, D. M., Tamaddon, H., Degenhardt, K., Sassoon, D., Morley, G. E., Jalife, J. and Fishman, G. I. (2001) Visualization and functional characterization of the developing murine cardiac conduction system. Development 128; 17851792.

34. Sasyniuk, B. I. and Mendez, C. (1971) A mechanism for reentry in canine ventricular tissue. Circ. Res. 28; 3-15.

35. Scher, A. M. and Spach, M. S. (1979) Cardiac depolarization and repolarization and the electrocardiogram. In "Handbook of Physiology, The Cardiovascular System, Vol I, Sec 2, The Heart", ed. by R. M. Berne, N. Sperelakis and S. R. Geiger, American Physiological Society, Washington, D.C., pp. 357-392.

36. Shimada, T., Nakamura, M. and Notohara, A. (1984) The Purkinje fiber-myocardial cell region in the goat heart as studied by combined scanning electron microscopy and chemical digestion. Experientia 40; 849-850.

37. Simon, A. M., Goodenough, D. A., and Paul, D. L. (1998) Mice lacking connexin40 have cardiac conduction abnormalities characteristic of atrioventricular and bundle branch block. Curr. Biol. 8; 295-298.

38. Sommer, J. R. and Johnson, E. A. (1968) Cardiac muscle. A comparative study of Purkinje fibers and ventricular fibers. J. Cell Biol. 36; 497-526.

39. Spach, M. S. and Kootsey, J. M. (1983) The nature of electrical propagation in cardiac muscle. Am. J. Physiol. 244; H3-H22.

40. Takamatsu, T. and Fujita, S. (1988) Microscopic tomography by laser scanning microscopy and its three-dimensional resolution. J. Microsc. 149; 167-174.

41. Takamatsu, T. and Wier, W. G. (1990) Calcium waves in mammalian heart: quantification of origin, magnitude, waveform, and velocity. FASEB J. 4; 1519-1525.

42. Tamaddon, H. S., Vaidya, D., Simon, A. M., Paul, D. L., Jalife, J. and Morley, G. E. (2000) High-resolution optical mapping of the right bundle branch in connexin 40 knockout mice revealed slow conduction in the specialized conduction system. Circ. Res. 87; 929-936.

43. Tanaka, H., Oyamada, M., Tsujii, E., Nakajo, T. and Takamatsu, T. (2002) Excitation-dependent intracellular $\mathrm{Ca}^{2+}$ waves at the epicardial border zone of cryo-injured rat heart revealed by in situ real-time confocal microscopy. J. Mol. Cell. Cardiol. 34; 15011512.

44. Tanaka, H. and Takamatsu, T. (2003) Spatiotemporal visualization of intracellular $\mathrm{Ca}^{2+}$ in living heart muscle cells viewed by confocal laser scanning microscopy. Acta Histochem. Cytochem. $36 ; 193-204$.

45. Tawara, S. (1906) Das Reizleitungssystem des Saugetierherzens. Gustav Fischer, Jena, Germany.

46. Toshimori, H., Toshimori, K., Oura, C., Matsuo, H. and Matsukura, S. (1988) Immunohistochemical identification of Purkinje fibers and transitional cells in a terminal portion of the impulseconducting system of porcine heart. Cell Tissue Res. 253; 47-53.

47. Tranum-Jensen, J., Wilde, A. A. M., Vermeulen, J. T. and Janse, M. J. (1991) Morphology of electrophysiologically identified junctions between Purkinje fibers and ventricular muscle in rabbit and pig heart. Circ. Res. 69; 429-437.

48. Truex, R. C., Curry, J. L. and Smythe, M. Q. (1954) Visualization of the Purkinje network of the beef heart. Anat. Rec. 118; 723735.

49. Valiunas, V., Weingart, R. and Brink, P. R. (2000) Formation of heterotypic gap junction channels by connexin 40 and 43. Circ. Res. 86; e42-e49.

50. Veenstra, R. D., Joyner, R. W. and Rawling, D. A. (1984) Purkinje and ventricular activation sequences of canine papillary muscle. Circ. Res. 54; 500-515.

51. Viragh, S. and Challice, C. E. (1973) The impulse generation and conduction system of the heart. In "Ultrastructure of the Mammalian Heart", ed. by C. E. Challice and S. Viragh, Academic Press, New York and London, pp. 43-89.

52. Viragh, S., Stoeckel, M. E. and Porte, A. (1987) Light and electron microscopic structure of the cardiac Purkinje fibers-review. Physiol. Bohemoslov. 36; 233-242.

53. Weiss, J. and Shine, K. I. (1982) $\left[\mathrm{K}^{+}\right]_{\mathrm{o}}$ accumulation and electrophysiological alterations during early myocardial ischemia. $\mathrm{Am}$. J. Physiol. 243; H318-H327.

54. Yater, W. M., Osterberg, A. E. and Hefke, H. W. (1930) Chemical determination of the glycogen ratio in the bundle of His and the cardiac muscle in man and in the horse. Arch. Int. Med. 45; $760-771$. 